\title{
Facilitating Collocated Crowdsourcing on Situated Displays
}

\begin{abstract}
Online crowdsourcing enables the distribution of work to a global labor force as small and often repetitive tasks. Recently, situated crowdsourcing has emerged as a complementary enabler to elicit labor in specific locations and from specific crowds. Teamwork in online crowdsourcing has been recently shown to increase quality of output, but teamwork in situated crowdsourcing remains unexplored. We set to fill this gap. We present a generic crowdsourcing platform that supports situated teamwork and provide experiences from a laboratory study that focused on comparing traditional online crowdsourcing to situated team-based crowdsourcing. We built a crowdsourcing desk that hosts three networked terminal displays. The displays run our custom team-driven crowdsourcing platform that was to investigate collocated crowdsourcing in small teams. In addition to analyzing quantitative data, we provide findings based on questionnaires, interviews, and observations. We highlight 1) emerging differences between traditional and collocated crowdsourcing, 2) the collaboration strategies that teams exhibited in collocated crowdsourcing, and 3) that a priori team familiarity does not significantly affect collocated interaction in crowdsourcing. The approach we introduce is a novel multi-display crowdsourcing setup that supports collocated labor teams and along with the reported study contributes particularly to situated crowdsourcing research.
\end{abstract}

Simo Hosio, Jorge Goncalves, Niels van Berkel, Simon Klakegg, Shin'Ichi Konomi \& Vassilis Kostakos (2018) Facilitating Collocated Crowdsourcing on Situated Displays, Human-Computer Interaction, 33:5-6, 335-371, DOI: 10.1080/07370024.2017.1344126 


\section{CONTENTS}

1. INTRODUCTION

2. RELATED WORK

2.1. Situated Technologies

2.2. Collocated Interaction and Teamwork

2.3. Situated Crowdsourcing

3. TEAMSOURCER

3.1. Crowdsourcing Desk

3.2. Android Client Application

User Interface

Moderation

Server Side

4. STUDY DETAILS

4.1. Tasks

T1: Identify Suspicious Elements

T2: Where's Waldo?

T3: Kinship Classification

4.2. Recruitment and Team Formation

4.3. Experimental Design

Methodology

Counterbalancing and Randomizing Task Order

4.4. Rewards

4.5. Tasks

5. RESULTS AND FINDINGS

5.1. Task Completion Efficiency

5.2. Qualitative Data

Contrasting Collocated and Traditional Crowdsourcing

Task Strategies Enabled by Collocated Interaction

Impact of Team Familiarity

Design Opportunities

6. DISCUSSION

6.1. Benefits of Collocated Interaction in Crowdsourcing

6.2. Challenges in Teamwork

6.3. Does Team Familiarity Affect Output?

6.4. Research Opportunities

6.5. Limitations

7. CONCLUSION

8. REFERENCES 


\section{INTRODUCTION}

Online crowdsourcing has been established as a means to complete computationally challenging tasks using a distributed, efficient, and cost-effective human labor force. Crowdsourced tasks are typically fast to complete and repetitive in nature, but also increasingly more challenging and creative tasks are being pitched to the crowds (Kittur et al., 2013; Morris et al., 2012).

One promising research direction is situated crowdsourcing: an approach to crowdsourcing that considers requesting and completing tasks inside geo-fenced boundaries. Situated crowdsourcing has rapidly gained popularity within academia, and the de facto means of enforcing situatedness is using location-aware mobile technologies (Alt et al., 2010). Here, instead of mobile phones, we enforce collocated crowdsourcing by using kiosk-style terminals that are mounted on a traditional physical table. The terminals run our custom crowdsourcing platform, that forms teams consisting of the people using the terminals. Thus, our study sets to explore collocated teamwork in a crowdsourcing context.

Prior work has contrasted traditional, online-based crowdsourcing to either online teamwork (Niloufar et al., 2017; Retelny et al., 2014) or situated solo workers (Goncalves et al., 2015; Hosio et al., 2014). Contrasting it to workers who are both situated and organized for teamwork, however, remains practically unexplored. This is the gap our work sets to fill (Figure 1). We admit that framing exact hypotheses for a study such as ours may be challenging. However, there exists ample evidence for anticipating how crowdsourcing in small teams may produce different type of labor and have distinct characteristics when compared to the much investigated subject of situated solo work (see e.g. (Goncalves et al., 2015; Hosio et al., 2014)). Further, enabling natural collocated interaction has been shown as beneficial in the contexts of e.g. sensemaking and brainstorming (e.g. (Lucero et al., 2010; Wozniak et al., 2016)), suggesting it may prove as useful in crowdsourcing as well.

Online

Traditional crowdsourcing (Kittur et al., 2013; Morris et al., 2012).

Team
Online teams (Niloufar et al., 2017; Retelny et al., 2014)
Situated

Situated worker (Goncalves et al., 2015; Hosio et al., 2014)

This work

\section{Figure 1. Previous work has explored teamwork and situated aspects of crowdsourcing, but not simultaneously.}

In one of the few reported studies that discuss aspects of situated crowdsourcing in teams, Goncalves et al. found workers perform sub-optimally when they are in the presence of friends or a group (Goncalves et al., 2013). In their study, however, the tasks and the interaction were not specifically designed for collocated collaboration, and did not provide appropriate scaffolding to support collocated interaction between people. This could have had an impact on the collected contributions. On the other hand, prior work also suggests that collaboration with friends is one of the core motives for completing situated tasks, particularly when workers can work towards a common goal, such as a shared reward (Hosio et al., 2014). This would suggest that it can be beneficial to design 
technological interventions for multiple collocated users to participate for the greater good, and that this area of research warrants further exploration.

For our study, we designed a 60-centimeter circular "crowdsourcing desk" (Figure 2) that hosts three terminal-sized display kiosks, each mounted at 120 degrees apart along the perimeter. Thus, each worker can converse and interact in other natural ways with the collocated workers. The kiosks consist of Android tablets inside metal enclosures. The tablets are configured so that users can only access our crowdsourcing application. Leveraging the described setup, we investigated how collocated workers complete different types of tasks together and what it means exactly to work as a collocated team in situated crowdsourcing. The tasks varied in complexity and were designed to be completed either alone or in teams. This work addresses the following research questions:

RQ1: How does collocated crowdsourcing differ from traditional online work from the worker's perspective?

RQ2: What type of strategies emerge when collocated teams complete crowdsourcing tasks?

RQ3: How do a priori social relationships between team members affect interaction and teamwork in collocated crowdsourcing?
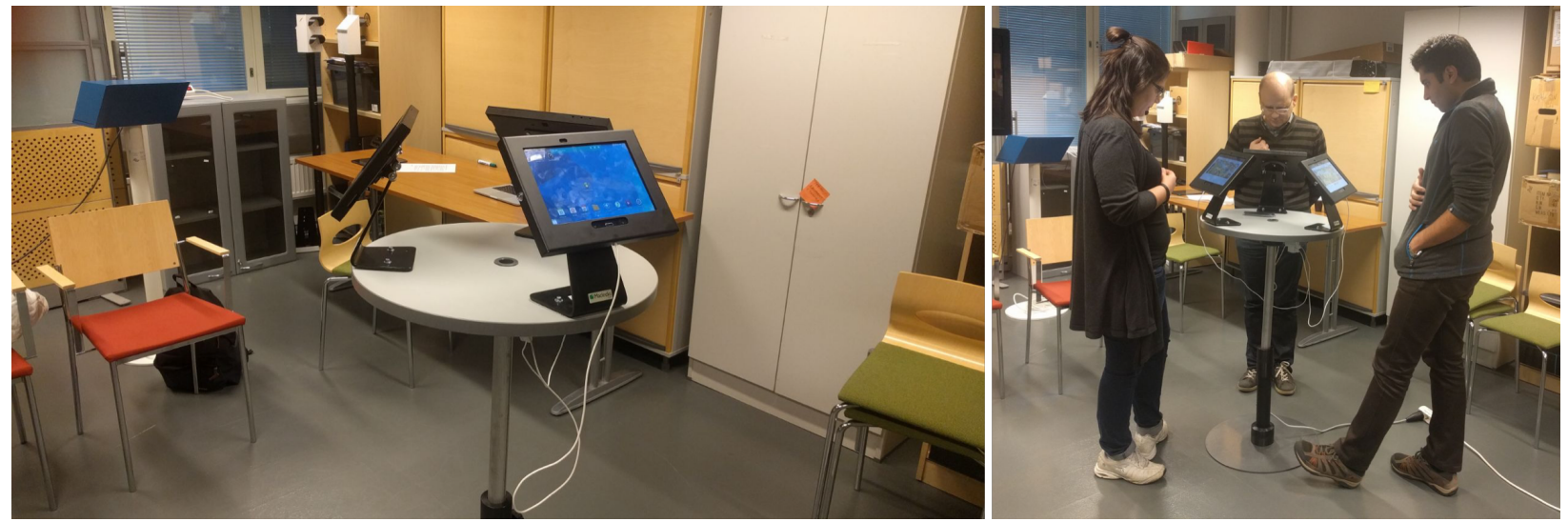

Figure 2: Our deployment in a laboratory setting, and a group working together on a task.

Our main contributions are derived from a qualitative analysis using questionnaires, interviews, and observations. Our analysis reveals that teams can complete certain types of visual search tasks faster than solo workers, and that teams complete subjective tasks with more in-depth consideration. We discuss how team strategies emerge before and during the actual work, and find that team familiarity affects collocated interaction, but in this case was not found to greatly affect task output. Our work extends the existing literature on situated crowdsourcing by proposing and investigating a relatively unexplored type of crowdsourcing system that physically embraces collocated interaction between workers.

\section{RELATED WORK}

Our study and setup are contextualized amidst three HCI research domains: situated technologies in general, collocated interaction and situated crowdsourcing. The three areas share similar research challenges and directions. Here we discuss the investigations and research that have motivated and directed our work. 


\subsection{Situated Technologies}

The overarching vision of ubiquitous computing is enabling computing everywhere to support our daily lives on the go. While this development perhaps most visibly manifests itself in the modern smartphones that today are ubiquitous devices in many parts of the world, researchers also explore how to make computing an integral part of the situated experience in our built environments (Greenfield \& Shepard, 2007). Such situated technology can be anything from passive sensors observing the environment to large interactive installations that provoke passersby to interact, either for levity or for more serious activities.

Our work is inspired by situated devices that facilitate purposeful interaction via any type of interfaces usable by the general public: walk-up-and-use systems. Essentially, for a public situated technology installation to genuinely serve the passersby, it needs to be so simple that first-time users can simply start using it without any external guidance (Brignull \& Rogers, 2003). Gesture control (Fikkert et al., 2009), Bluetooth (José et al., 2008), or using mobile phones as proverbial remote controllers (Boring et al., 2009; Hosio et al., 2010) are examples of how interaction with situated technologies can be facilitated. However, perhaps the most straightforward approach to interact with situated technologies is by touch interaction. Touch screens are becoming increasingly reliable and affordable, and thanks to the development of smartphones, users are becoming very skillful and comfortable with touch-based interaction. There exist numerous sophisticated situated display deployments and studies that explore how users encounter and interact with situated deployments, e.g., (Hosio et al., 2016; Memarovic et al., 2012; Müller et al., 2010; Storz et al., 2006), even in the crowdsourcing domain alone (Goncalves et al., 2013; Heimerl et al., 2012; Hosio et al., 2014).

The situated technology we evaluate in this paper is most closely related to public displays. Situated displays have a decades-long history of research and practice behind them. For instance, one of the best known historic display deployment, Hole-In-Space, by Kit Galloway and Sherrie Rabinowitz, used situated screens and audio to connect the people of New York City and Los Angeles already in 1980 ("Hole-In-Space," 2016). Dubbed playfully as "the mother of all video chats", the installation concretely demonstrated how versatile situated display installations can be when placed in contextually suitable locations and in front of the right audience. Since then, a series of well-known experiments at Xerox PARC explored using situated displays in the office settings using several different sizes of displays (Bly et al., 1993; Weiser, 1999). Recently, researchers have also started to explore the possibilities afforded by façade-sized projector-based screens (Gehring \& Krüger, 2012), and some even compare the societal potential of public displays to that of radio, television, and the Internet (Davies et al., 2012).

A key human characteristic that situated technologies, such as displays, can exploit is the fundamental desire to explore, to start using pieces of technology rather serendipitously and simply to "kill time" (Müller et al., 2010). Thus, situating the deployment at locations where people typically have free time is considered beneficial. The powerful visual capabilities of displays allow for rich types of content to be offered to users (see e.g., (Ojala et al., 2012; Storz et al., 2006)). In addition to placement and content, also the potential audience must be taken into consideration for sweet spots to emerge, where meaningful connections with users are likely to take place with public displays (Schroeter et al., 2012), or indeed with any type of situated technology. The main takeaway here is that public displays as visual and interactive elements can be extremely flexible and compelling platforms for researchers and application developers. Crowdsourcing on public displays is becoming increasingly 
feasible as the number of display installations grows, and with our work we seek to introduce the first public display crowdsourcing platform that supports collocated teamwork.

Naturally, situated technologies also offer specific challenges. For example, researchers have identified and tried to alleviate barriers of interaction with displays (Brignull \& Rogers, 2003; Hosio et al., 2016; Kukka et al., 2013). Another real-world challenge with public displays is their strenuous maintenance and the constant need to display fresh content (Storz et al., 2006). Another problem, as well as an opportunity, is reliable runtime assembly of multi-device ecologies (Hosio et al., 2010; Weißker et al., 2016). Other promising use cases for displays are civic engagement (Hosio et al., 2012), artistic experiences (Scheible \& Ojala, 2009), or fostering local communities (Memarovic et al., 2013; Taylor \& Cheverst, 2012). Examples of long-term research projects with larger situated display and technology networks are the European Union project PD-NET with their "Networked Public Displays" (Memarovic et al., 2016), the UBI-hotspots in Oulu, Finland (Hosio et al., 2016), or the Wray display installation in the UK (Taylor \& Cheverst, 2012). Such larger projects have contributed especially in documenting the interaction with local authorities and challenges of making the installations a permanent part of their surroundings.

However, when introducing technology as a new work or communication medium, social cues and nuances are lost (Desanctis \& Gallupe, 1987). We crafted our situated setup -- an ensemble of kiosksized public displays -- so that small teams can simultaneously complete crowdsourcing tasks while interacting as naturally as possible with each other. Hence, our work presents an insightful first look into the affordances, issues, and possibilities emerging in such collocated interaction and situated teamwork in the context of crowdsourcing.

\subsection{Collocated Interaction and Teamwork}

In everyday settings humans naturally take into consideration the interactions and actions of nearby people. Working in a collocated fashion has been studied extensively in HCI and CSCW communities, and in general users tend to find cooperation as somehow more fun and engaging than working alone (e.g. Porcheron et al., 2016; Lucero et al., 2010). However, our emotions and biases lead to conflicts between rationality and irrationality in team situations, where the individual has to choose between self and collective interests (Kagel \& Roth, 1995). Game theorists and behavioral economists have been studying team behavior for decades, identifying e.g. phenotypes for individuals in cooperative gaming (Optimist, Pessimist, Envious, Trustful) (Poncela-Casasnovas et al., 2016). Further, ethnographic work on observing disaster response teams highlighted the importance of recognizing and pre-emptively dealing with uncertainties in field deployments (Fischer et al., 2015). This is particularly relevant to HCI and UbiComp deployments in general, as real-world deployments are riddled with uncertainties and unforeseeable issues that researchers must deal with (Huang et al., 2007; Storz et al., 2006).

Situated technology deployments are often used by groups of users (Hosio et al., 2012). However, while using technologies in groups of people is fun and entices interaction, social awkwardness has also been documented in such situations. For example, the space around a public deployment can be perceived as a proverbial stage where the audience is watching the user (Kuikkaniemi et al., 2011; Müller et al., 2010). The group members also sometimes conflict with each other when using a shared deployment. For example, Peltonen et al. studied social group interactions in an urban city area using a

public display as an intervention (Peltonen et al., 2008). They document in detail how the presence of 
users invited new interactions to take place with the deployment, and how the presence of others often leads to conflicts and tensions in the personal spaces of users.

The presence of users who are publicly interacting with a deployment draws the attention of passersby - a phenomenon better known as the honey-pot effect (Brignull \& Rogers, 2003). The honeypot effect can be leveraged to increase interactions with a deployment simply by designing for attention and affording also the audience to start using the deployment (Hosio et al., 2012; Müller et al., 2014). However, deployments often tend to support only one user at a time, and therefore the honey-pot effect leads to queueing. Here, we are interested in situations where collocated interaction takes place in a group or in a team with a shared goal. Examples include playful community applications, where people tailor or create the content on display (Farnham et al., 2009; Hosio et al., 2010; Scheible \& Ojala, 2009). In addition to mobile devices, previous work has used traditional and digital tabletops (Fischer et al., 2015; Scott et al., 2004), wearable devices, and combinations of all these for a plethora of different purposes. A compact and timely overview of the related research directions can be found in (Anslow et al., 2015).

Teamwork can occur without the use of situated technologies, such as mobile devices that are just used together. The MindMap, by Lucero et al. (Lucero et al., 2010), for example, demonstrated how users can collaboratively create a mind map with mobile devices, and how such collocated co-creation fostered engagement between the participants. Further, The Internet has enabled assembling teams using computational systems and coordinating even complex teamwork without any limiting spatiotemporal boundaries. For instance, using Etherpad (a lightweight collaborative online notepad), workers from MTurk have been successfully tasked with translating Spanish poems into English (Kittur, 2010). Flash Teams is a framework to coordinate experts from a crowd to perform e.g. rapid design prototyping or course development (Retelny et al., 2014). The work with Flash Teams also highlights how it is possible to create entire organizations consisting of teams with different skillsets and that can practically provide output 24 hours per day, as the workforce is truly global. Yet another example is Huddler that is used to assemble familiar teams during uncertain availability from MTurk. Huddler (Niloufar et al., 2017) provides a thin wrapper where workers wait for other workers to join the ad-hoc team before proceeding to complete the actual tasks. Taking the concept even further, Team Dating grants the power to assemble efficient teams to the workers (Lykourentzou et al., 2017). MTurk workers, or Turkers, as they have dubbed themselves, have also developed elaborate ways on their own to collaborate in identifying lucrative tasks and in recreating the social aspects that exist in traditional brick and mortar work (Gray et al., 2016).

Finally, sensemaking is related to our work, as it is concerned with collecting, organizing and presenting information about a problem that needs to be solved. Design choices and goals similar to our work can be found, for example, in RAMPARTS that assembled mobile devices to aid small teams in collocated problem solving (Wozniak et al., 2016). With RAMPARTS, task completion times were reduced in comparison to a traditional (in this case: paper based) approach while users' cognitive load did not increase. Another example from the sensemaking domain is $S A V A N T$, used e.g. for distributed collaboration on a realistic task of identifying a serial killer (Goyal \& Fussell, 2016). The study highlights how a sensemaking system should support translucency and bidirectional, realtime information sharing to reduce confirmation bias and to make the entire process more efficient for the end users. 
Our work strictly considers groups working closely together in situated settings. Group familiarity has been identified as a positive factor affecting work quality, and is why Huddler successfully brings together familiar online workers (Niloufar et al., 2017). In offline settings, familiarity boosts a team's performance by enabling more efficient coordination than in teams with unfamiliar members (Reagans et al., 2005). With our deployment we set to investigate how the relationships inside a team may affect interaction and thus output. Further, and given that we are dealing with situated crowdsourcing where solo workers have been documented to contribute higher quality input than groups (Goncalves et al., 2013), we set to discuss how to best leverage the emerging defining characteristics of teamwork in collocated crowdsourcing.

\subsection{Situated Crowdsourcing}

Situated crowdsourcing has recently emerged as a complementary method of leveraging the crowds for different types of tasks. Situated crowdsourcing is typically facilitated by using mobile technologies (Alt et al., 2010; "Clickworker," 2014; Hosio et al., 2016; Sasao et al., 2016), but is being increasingly explored by leveraging the specific beneficial characteristics of situated technologies that were discussed in the previous section. Using situated deployments that are built as parts of our everyday environments enables a low barrier of entry for people who would not be otherwise reachable as well as efficient targeting of wanted participants (Goncalves et al., 2013; Hosio et al., 2014).

Examples of recent notable situated crowdsourcing deployments include an augmented vending machine, Umati (Heimerl et al., 2012), that facilitated communitysourcing at a university campus. Using a touchscreen as the input device, it offered non-monetary rewards (snack items) in exchange for labor. They demonstrated how situated crowdsourcing can provide better accuracy than traditional online crowdsourcing when the tasks requires local and specialized knowledge. A larger-scale deployment, Bazaar, investigated how a situated labor market that follows a market model can be built using small kiosk-sized public displays (Hosio et al., 2014). Bazaar supports arbitrary tasks and rewards, and has for example been used to collect structured data on subjective questions (Goncalves et al., 2017). Recent ongoing work has also introduced CrowdButton and CrowdFeedBack to explore the sustainability of unpaid situated crowdsourcing contributions and improving contribution quality (Huang, 2015). Finally, Ludwig et al. presented an empirical study on coordination between volunteers and emergency personnel (Ludwig et al., 2016). The introduced public display application, City-Share, demonstrated how situated crowdsourcing can help managing the activities of different stakeholders on the disaster zone as well as enhance situational awareness.

A typical criticism of situated crowdsourcing is its limited scale and reach. Indeed, online crowdsourcing has the potential to reach billions of users (Ipeirotis \& Gabrilovich, 2014). However, situated crowdsourcing should be seen as a complementary means instead of a replacement of traditional crowdsourcing (Hosio et al., 2014). Aspects such as worker performance and the meaning of work (e.g., fun, camaraderie, as suggested in (Kittur, 2010)), payment schemes, etc. differ across markets and environments. Thus, and because existing situated crowdsourcing studies show promise on several fronts, learning as much as possible about different types of future crowd work setups is warranted. Next, we describe our contribution to situated crowdsourcing: TeamSourcer. 


\section{TEAMSOURCER}

Here we describe the design and implementation of TeamSourcer -- an Android application designed to run on tablet devices and to support collocated crowdsourcing in groups of people, i.e., teams. Next we describe the most important features and functionality of our experimental setup.

\subsection{Crowdsourcing Desk}

For the purposes of the study, we assembled a custom-designed desk that hosts three Android tablets inside metal enclosures (see Figure 2). The desk's height is adjustable and the diameter of the wooden surface is $60 \mathrm{~cm}$. The tablets are facing outward from the table's center, mounted at 120 degrees from each other. We opted for a standing desk to maximize the chances of new users coming and exploring the system serendipitously, just like interactions with public displays often start (Müller et al., 2010), instead of potential users simply using it as a desk for longer periods of time. Our setup is by definition a few-to-few, coupled display ecosystem (Terrenghi et al., 2009) that allows for natural interaction between users to occur. Being able to discuss and interact naturally has been found as essential when coordinating shared work on mobile devices in a highly similar setting to ours (Porcheron et al., 2016). Further, a similar design was used with e.g. with UbiTable to support easy access to the deployment and impromptu face-to-face collaborations for small groups of people that (Shen et al., 2003). Around our desk, the users can easily communicate with each other, but do not see each other's screens without explicitly moving aside to peek. This type of setup facilitates collocated work in a "public space" while also offering the workers a private "working space" (Shen et al., 2003). The setup can be also be easily installed in new locations, as only power and Wi-Fi are required. While the setup supports our research goals, the design choice also aims to avoid collisions that occur when collocated people work on the same screen and in the same space (Peltonen et al., 2008).

\subsection{Android Client Application}

The Android client application, TeamSourcer, supports both traditional situated crowdsourcing, where one worker completes tasks, and tasks that require two or more team members to be simultaneously logged in to the application. It is not limited to use with the described desk setup. However, in this case the number of simultaneous users was practically limited to three since we deployed TeamSourcer on the aforementioned crowdsourcing desk. TeamSourcer teams earn credits by completing tasks deployed in the system, and the credits are attributed to the whole team instead of individual workers. To keep the system flexible, the protocol for exchanging credits to monetary or other rewards is not fixed by design and can be adjusted on a per-study basis. This is explained next, along with all the key features currently implemented in the application.

\section{User Interface}

The user interface of TeamSourcer provides six main screens that can be seen in Figure 3. TeamSourcer's visual design and functionality is influenced by Bazaar - a situated crowdsourcing market for solo workers (Hosio et al., 2014). Bazaar has been shown easy to use and functional, and thus we recreated much of the functionality and added features to support not only solo workers but also teams of arbitrary size. Each screen in TeamSourcer implements one of the six main functionalities described next. 
1) Create Team or Login. The default screen that opens when launching the application, Create Team or Login, invites potential workers to either create a new team by entering a unique team name and a password, or to login using existing credentials that were created earlier. The user also has to provide her own email address, so that the system can keep track of individual users belonging to a team. If the email address is not found in the application's user table a new entry is created. The left side of the screen provides concise information about the application.

The worker who initiates a session by logging in or creating a new team in this screen automatically becomes the responsible leader for the session. A session lasts until the leader logs out from the application by returning back to this screen. In TeamSourcer, the leader is the only worker in a session who can submit a task. The decision of having a team leader instead of letting everyone submit tasks is informed by work on Flash Teams (Retelny et al., 2014). In Flash Teams, responsible leaders are appointed by the system in runtime to ensure high quality task completion and to coordinate the work. We, on the other hand, let users become a leader by taking initiative and starting a work session.

2) Join Session. When any of the TeamSourcer instances is logged in using the previous screen, a work session starts, and the two other tablets automatically transition to the Join Session screen. The state change is communicated between the instances using Google Firebase Messaging (previously Google Cloud Messaging). Here, the user can only join the ongoing session, and is only asked for the password, as the team id is communicated between the tablets automatically. Again, the user's own email acts as the individual user's unique identifier, and a new user is created as a member of the logged in team if the provided email address has not been previously used with TeamSourcer.

3) Home Screen. Home Screen displays the team's current credit balance (combined credits earned by all team members). Further, links to Task Gallery and Storefront are displayed.

4) Task Gallery. Task Gallery displays all currently available tasks in TeamSourcer. The tasks are dynamically fetched from the back-end database upon launching the activity. The availability of a task can be limited by how many simultaneous team members are needed to be logged in at the moment. The task itself is required to implement any restrictions of how many instances of each task a team or an individual member is allowed to complete. This is explained later when we describe the tasks and task mechanisms used in this study in more detail.

5) Storefront. Storefront displays instructions on how to claim rewards and what these rewards actually are. As we did not wish to limit the reward scheme, the rewards can be anything the administrative team wants to offer, and the screen simply embeds an instructional web page. In this study, the page consists of an image and a short text stating that the study reward per each worker is a movie ticket that is paid after successfully participating in all study stages.

6) Task Screen. Finally, Task Screen is where workers complete tasks. The task screen is opened from the Task Gallery, and it embeds in a WebView an external URL pointing to a task location. The page receives as HTTP GET parameters the following information from TeamSourcer:

- userId: the worker id that is currently using the TeamSourcer application launching this task 
- teamId: the team id that the current user belongs to

- isLeader: either 1 or 0 , depending on if the user is the current leader or not

These three parameters can be used to limit the task instances that a team or an individual worker can complete. For example, in a typical case each worker, or in this case a team, is allowed to complete each task exactly once, or until the pool of tasks run out for that user.

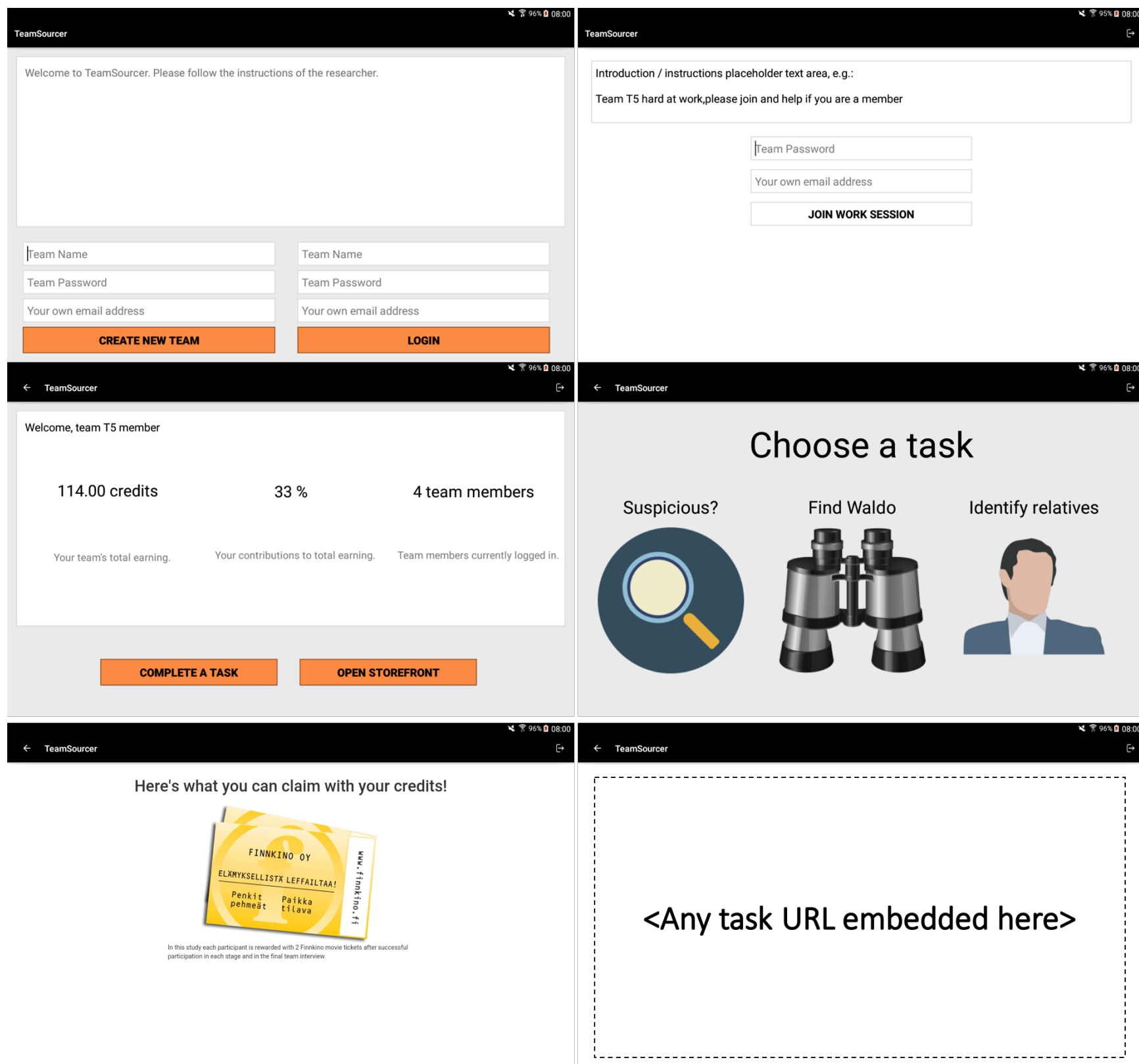

Figure 3: Screenshots of TeamSourcer. Top row: Create Team or Login, Join Session. Middle row: Home Screen, Task Gallery. Bottom row: Storefront, Task Screen (the embedded task omitted from the screenshot). 
Using Android's own callback functionality, the tasks notify TeamSourcer on the completion of a task instance. The callback supports up to four sub-id parameters (S1-S4) that are relayed to the database by the TeamSourcer client. The sub-ids can be arbitrary strings, and it is the task owner's responsibility to specify these in such a way that all relevant data is captured during the crowdsourcing process. Should the task owner / designer so choose, the task is not required to pass anything in sub-ids, and the task can implement its own mechanisms to store results. However, the callback must still be called upon completion of each task instance in order to compensate workers for their labor. In addition to the sub-ids, the TeamSourcer client submits the current reward of the task, workerId and teamId to the database upon completion of a task instance.

\section{Moderation}

A key feature in crowdsourcing systems is moderation. TeamSourcer's data model contains a field for contribution quality (or disapproval). However, for this study we did not implement any moderation features, as the tasks were completed in a laboratory environment and for a fixed payment. Moderation of crowdsourcing tasks completed as teams is also a previously unexplored concept, and we see this as an avenue to explore with our future deployments with TeamSourcer.

\section{Server Side}

TeamSourcer's server side components are implemented in PHP + MySQL and handle all communication between the clients the underlying databases. Further, TeamSourcer communicates between other instances using Google's Firebase Cloud Messaging (FCM), and FCM-related tokens are stored in a database. Further details of the server side are out of the scope of this article.

\section{STUDY DETAILS}

\subsection{Tasks}

We conducted a study in our usability lab, where we invited workers to complete 3 different types of visual search tasks:

- T1: Identify Suspicious Elements. Participants have to indicate suspicious elements in photographs of public settings.

- T2: Where's Waldo? Participants have to find a particular character in a photo.

- T3: Kinship Classification. Participants have to indicate whether two individuals shown in separate photographs are biologically related.

Visual tasks where workers annotate or identify things in images are inspired by several popular online real-world crowdsourcing initiatives, such as TomNod and ZooniVerse (Smith et al., 2013; Tomnod, 2016). We implemented the experimental tasks by embedding a collaborative whiteboard application (AWW) (A Web Whiteboard, 2016) to a web page, loading image(s) on it, and controlling the canvas actions using its API and button elements on the task page. For our purposes, AWW offers a touch-optimized, customizable, and collaborative HTML5 canvas with elegant text and drawing tools. We provided a drawing marker (default drawing with red color) and an erase tool, which in practice 
leaves a white mark (we wanted to be able to see in the final results what people erased). We also built a specific server-side API endpoint, which could be called instead of the Android callback, so that the tasks could also be completed as "traditional" online tasks using a desktop web browser (each team member first completed the tasks alone). Each task included a short instruction that the participant was able to read prior and during completion of the task.

\section{T1: Identify Suspicious Elements}

In T1 workers were asked to identify any and all suspicious elements (people, items, things in general) in pictures taken from crowded spaces all around the world. This task is subjective and thus has no ground truth available to it. While this naturally hinders the analysis possibilities from the viewpoint of our study, it is a highly realistic task, as real-world crowdsourcing tasks often lack ground truth and the assessment is collected from the crowd (Hosio et al., 2016; Kittur, 2010). We embedded the AWW canvas on a page with short instructions that stated:

"Indicate (for example by drawing a circle, arrow or a dot) anything or anyone that you think is suspicious, dangerous or otherwise worth further inspection in the picture. There are no wrong answers, but you should be able to justify your choices, if asked."

T1 featured a single button to submit the current image. When completing the task as a team, only the leader could see the button. The images with the overlaid drawings were saved on our servers, and the next image was loaded from our image repository. In total T1 had 20 images we manually chose. Each participant annotated half of them alone, and the other half as a team. A screenshot of T1 and two examples of the source images can be seen in Figure 4.
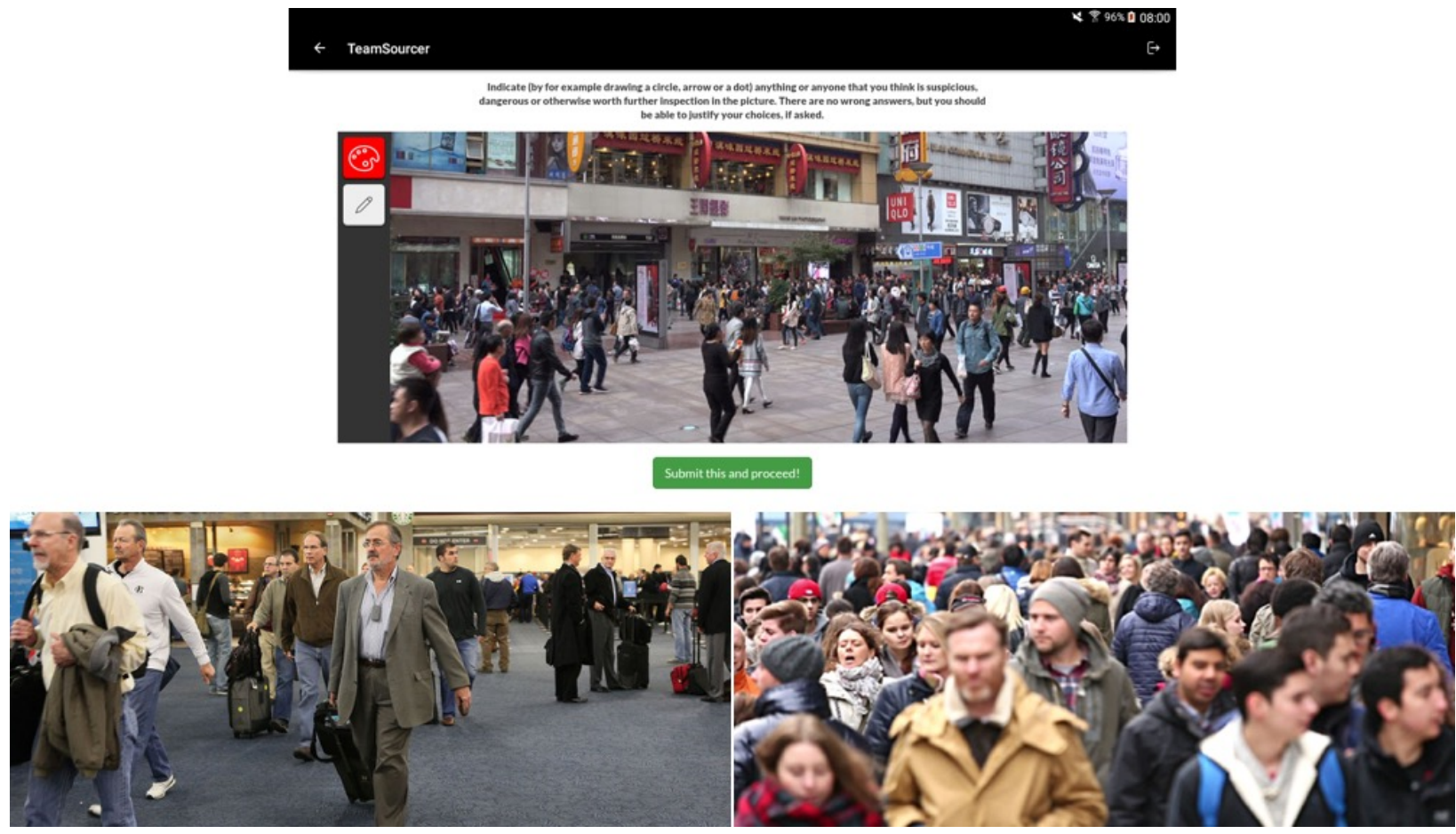

Figure 4. Top: A screenshot depicting T1, where users are asked to identify suspicious elements in the image. Bottom: two other images used in T1. 


\section{T2: Where's Waldo?}

T2 is the classic task of finding the fictional character "Waldo" (or Wally) in a cluttered image full of other characters. In our case the images were obtained from the official social media pages of Where's Waldo (Pinterest, Facebook). This task was designed to be competitive: the quickest individual participant as well as the quickest team were promised a bonus reward after the study was completed. The task also followed a similar layout to that of T1, and the instructions stated:

"Find Waldo, and mark him with a red circle. But be quick! We record your time, and the team that finds all Waldo's faster than others gets an extra reward! Also, the fastest individual worker will get an extra reward!"

The sense of urgency was further instilled by adding a stopwatch in the task below the canvas that kept track of how many seconds the individual / team had spent in the task so far. In T2 the control buttons also featured a skip-button in addition to a submit button. If a user or team simply could not find Waldo, they had the option to skip and move on to the next image.

For T2 we used 20 solvable Where's Waldo images, i.e., there was Waldo in each image. A screenshot of T2 and examples of the source images are depicted in Figure 5.
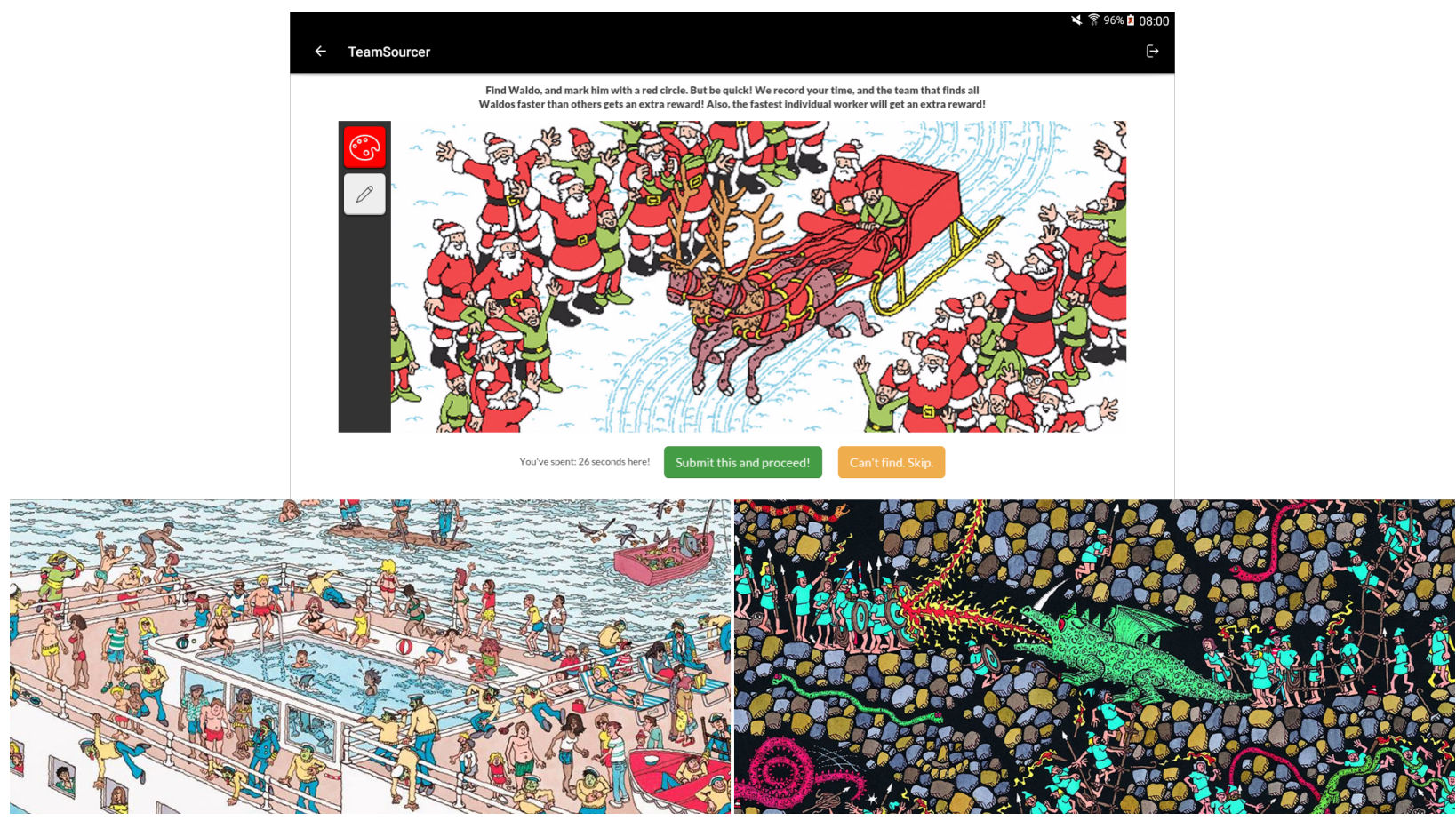

Figure 5. Top: A screenshot depicting T2, where users have to find Waldo. Bottom: two other images used in T2. 


\section{T3: Kinship Classification}

T3 was to identify whether two people are biologically related. The instructions on the top of the task page stated:

"Look at the photos of the 2 people below and give your assessment if you think they are related (i.e. part of the same family). Please keep in mind that the quality of your assessment will directly influence the quality of this research. In addition, mark the facial features you find similar, if any!"

The images and kinship ground truth were obtained from a research database (KinFaceW: Kinship Face in the Wild Database, 2016). We hypothesized that T3 would provoke a lot of collaboration and negotiation between the team members, and that therefore it would constitute a suitable task in regards to our study goals. T3 had 16 image pairs. In 8 of the image pairs the persons were relatives, and in 8 they were not. The controls in T3 consisted of a submit button and radio buttons to mark the pair as either related or not related. The radio buttons were reset to unchecked after each submission. No selection was required, so the participants could also submit without stating an opinion about kinship if they were undecided. T3 can be seen in Figure 6.

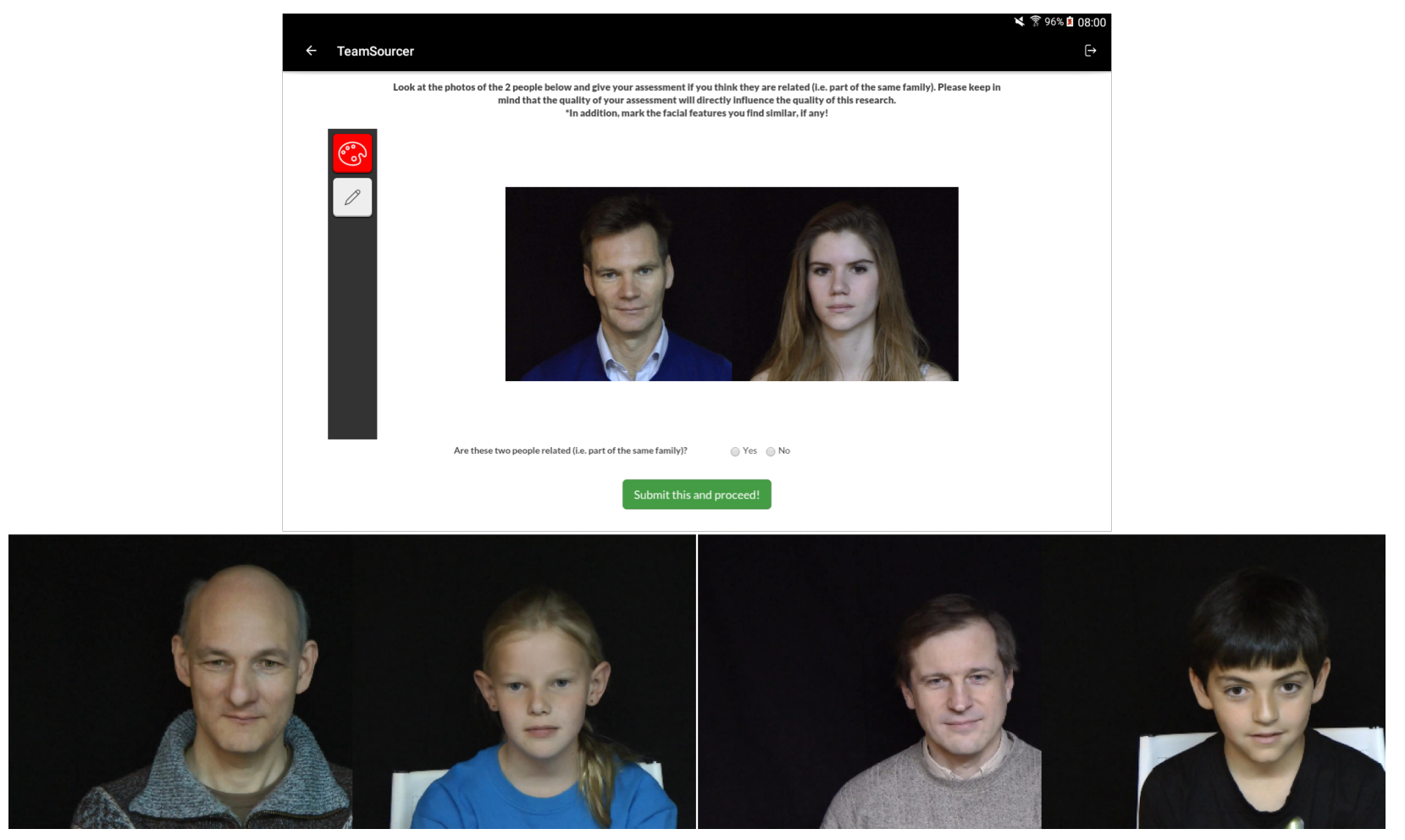

Figure 6. Top: A screenshot depicting T3, where users are asked to select if the persons are related and to mark similar facial features. Bottom: two other images used in T3.

\subsection{Recruitment and Team Formation}

We invited participants to the study using social media as well as selected email lists of students and staff in our university. In the call, we solicited for groups of three friends or single individuals, so 
that we could form teams where all members are friends, and teams where all members are strangers to each other.

We split the participants into 8 teams for the study. Each team had three members, and thus the study had 24 participants in total (13 male, 11 female, average age: 30.5, SD: 5.3). When forming the teams, we balanced the amount of teams consisting of friends and strangers, so that we ended up with 4 of each type of teams (friends / strangers). We also actively promoted participant diversity, and chose participants from several different study areas such as computer science, biomedical engineering, biology, education, and product management.

\subsection{Experimental Design}

Participants completed the study in groups of three members, as described in the previous section. Each group was asked to arrive to our laboratory premises, and upon arrival the entire team was first welcomed and briefed about the study in an office of one of the authors. Then, the participants were split and each individual member was asked to complete all experimental tasks using a PC provided by us in an isolated working space. This was done to give the participants an experience of how traditional crowdsourcing feels like and for us to get data on how the participants performed alone. Doing this, we hypothesized that participants would be better able to reflect on the differences between traditional crowdsourcing and the collocated work environment about to be introduced to them. Naturally, at this point the team members had no interaction with each other whatsoever, and no teamwork or collaboration happened.

After completing the tasks alone, the three participants were brought together and provided an introduction to the crowdsourcing desk and the TeamSourcer application (Figure 2). The team was then instructed to complete the experimental tasks again, but this time as a team. As explained earlier, the tasks that the team completed here consisted of new images that they had not seen earlier when completing them alone. This was done to mitigate any potential learning issues. Participants were instructed to act and work as they wish to, naturally, as a team sharing the same mission. After completing the tasks as a team, each participant completed a questionnaire with demographic data and open-ended questions about key points in the study:

- Gender, age, major study area

- Team members' familiarity with each other

- If strangers were present in the team, how did it affect interaction with the team or an individual member

- Biggest differences, exact pros and cons in crowdsourcing alone or in a collocated team

- What kind of work is suitable / not suitable for completing alone or in teams

- Thoughts and experiences on using TeamSourcer as a basis for the tasks

- Other improvement ideas for facilitating teamwork with TeamSourcer

Finally, after the participants completed the questionnaire, we conducted a final interview with the entire team together in the same office where they were initially briefed. The discussion focused mainly on exploring the strategies the solo workers and the teams employed in each task, but all other issues that the team wished to share were captured as well. 


\section{Methodology}

During the teamwork stage (after participants had completed the tasks alone), two researchers were unobtrusively observing the teamwork from a remote corner in the same office space and took notes of any interactions and discussions taking place around the table. The researchers did not offer any help in using the application itself or in completing the tasks.

In analysing the questionnaire responses and interview results, we followed an adapted version of $a$ priori coding approach (Weber, 1990). In practice, two of the manuscript authors together inspected the quotes and classified them based on which of the research questions can they provide answers to, if any. Then, instead of taking a subsample of the classified items (the original approach), two external validators inspected the classification, suggesting quotes to be appointed to different categories when necessary. In all disputed cases, the authors discussed with the external validators until a consensus was reached on the category the item belongs to. Similar analysis has been used to analyse open-ended text entries $e . g$. in (Hosio et al., 2015).

\section{Counterbalancing and Randomizing Task Order}

All participants completed the solo condition first (working alone), as described earlier, and then continued as teams (working as teams). Each of the three tasks contained two sets of images. A team was always allocated a different set for working alone and for working as a team. Thus, a participant never saw the same image twice. This mitigates any potential learning effects.

Further, the order of the sets in each task was counterbalanced so that half of the teams would see "set A" when working alone, and the other half of the teams would start with "set B" when working alone. Finally, in every case, the completion order of images in a set was randomized.

\subsection{Rewards}

The rewards were scaled so that upon completing the study each team would have enough credits for two movie tickets per team member. The 2 tickets together are worth $€ 20$. In situated crowdsourcing rewards can be higher than in traditional online environments, as cultural and local pay grade must be taken into consideration (Hosio et al., 2014). Given the average salary in <country>, where the study was run, the reward roughly equates to 1 hour of work, which matches the approximate duration of the study per participant.

\section{RESULTS AND FINDINGS}

\subsection{Task Completion Efficiency}

Figure 7 presents the average time and standard deviation for completing an instance of the three tasks T1, T2, and T3. The difference in completion times did not significantly differ between stranger and friend teams in any of the tasks. However, as determined by a one-way ANOVA, there is a statistically significant difference in completion time between solo workers and all teams (friend and stranger teams combined) in all tasks (T1 and T3: $\mathrm{p}<0.001, \mathrm{~T} 2: \mathrm{p}<0.01)$. Teams spent longer time in T1 (Identify Suspicious Elements) and T3 (Kinship Classification). As expected, teams were faster in T2 (Where's Waldo) (Figure 7). 
For T1 we analyzed the number of suspicious elements marked per picture, i.e., per each task instance. Teams (combined) marked an average of 1.6 elements whereas solo workers only 0.9 elements. Similarly, in T3, teams identified an average of 2.3 similar facial features per trial (pair of faces) whereas solo workers marked 1.6 features. A Wilcoxon rank-sum test was performed to successfully validate the significance of the observed differences in T3 ( $p<0.001)$.

In terms of accuracy, for T2 we analyzed how often teams and solo workers successfully completed the task, i.e., found and marked Waldo in the pictures. We found no significant differences: teams correctly found Waldo in $90 \%$ of the images, and solo workers in $94 \%$. Similarly, there was no significant difference in the accuracy between teams and solo workers in T3. Furthermore, familiarity did not affect accuracy in T2 or T3 (for T1, there is no ground truth and therefore no accuracy measure). Figures 8, 9, and 10 below depict result images from each of the three tasks.

\begin{tabular}{lccc} 
& T1 (SD) & T2 (SD) & T3 (SD) \\
Solo & $31.6(24.2)$ & $10.1(9.1)$ & $19.6(13.7)$ \\
Team (overall) & $56.6(30.0)$ & $7.0(6.5)$ & $29.1(16.2)$ \\
Team (friends) & $62.7(33.8)$ & $6.5(3.2)$ & $27.4(16.6)$ \\
Team (strangers) & $50.7(24.8)$ & $7.6(8.6)$ & $30.8(16.0)$ \\
\hline
\end{tabular}

Figure 7. Average time (seconds) spent per a single task instance across all three tasks when working alone or when working in teams.

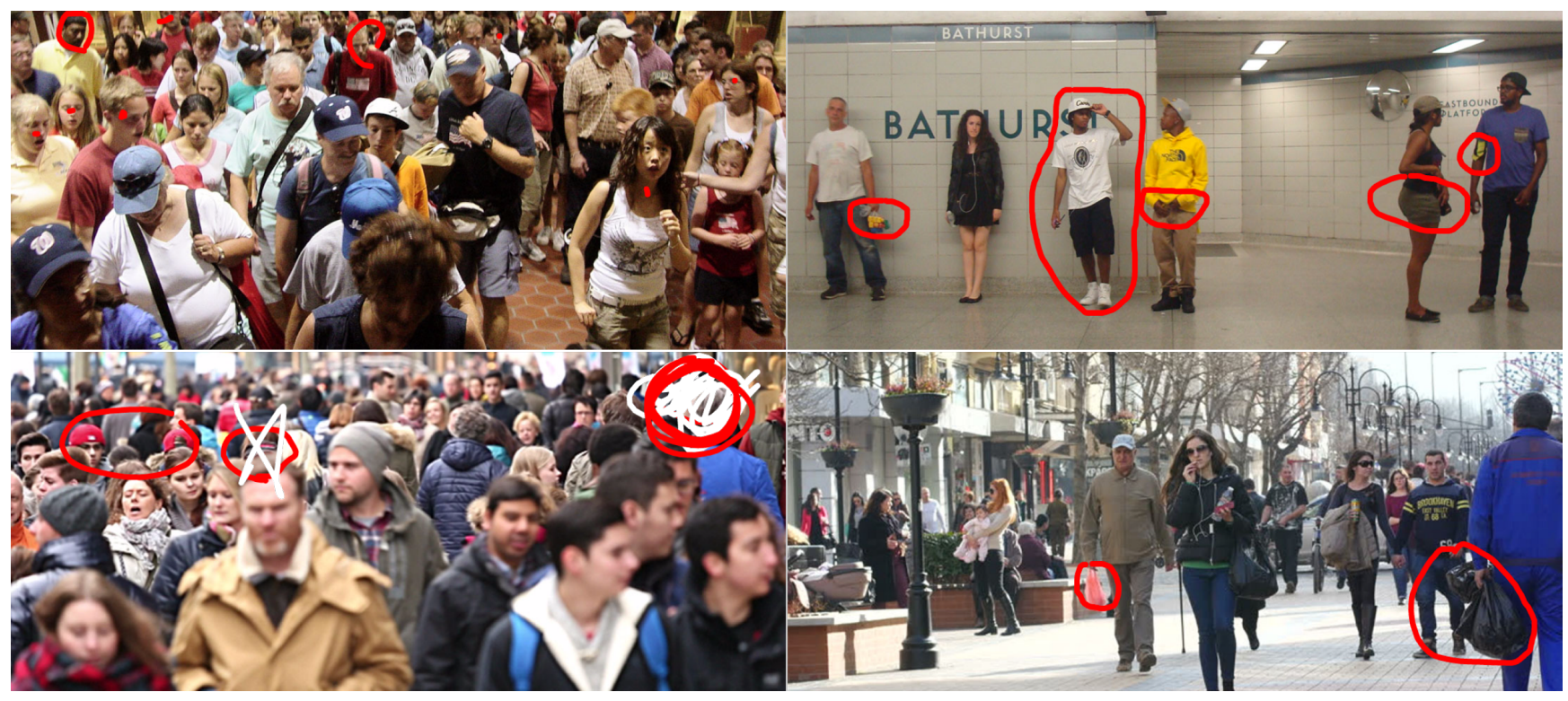

Figure 8. Examples of submitted images from T1: identify suspicious elements. White color denotes erased marks. 


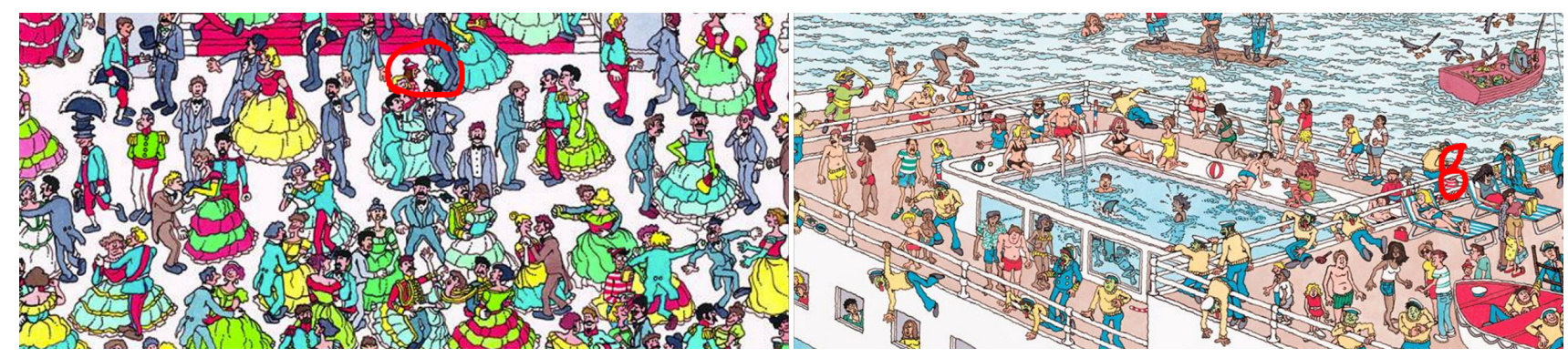

Figure 9. Examples of submitted images from T2: Where's Waldo?

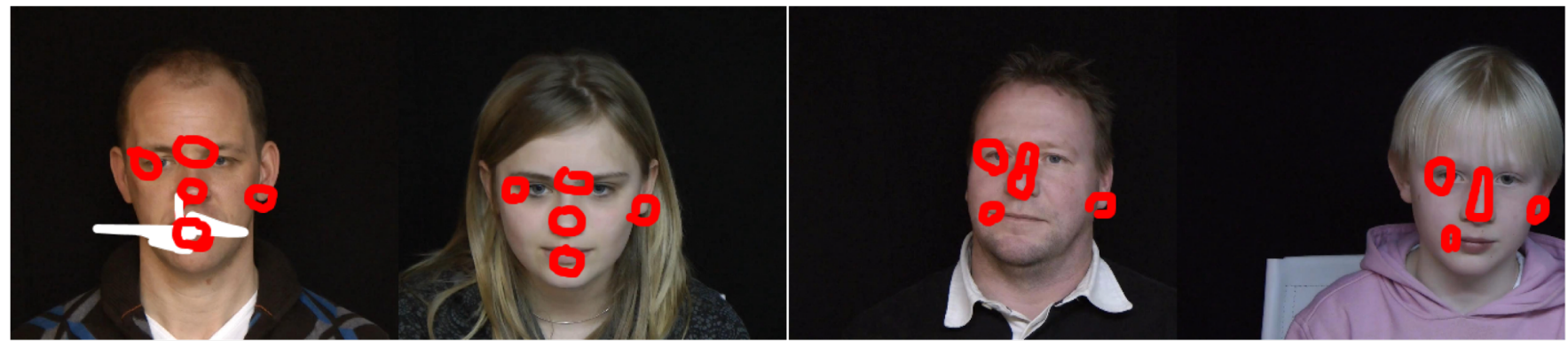

Figure 10. Examples of submitted images from T3: kinship. The white marker indicates an "erased" entry, as a result of debating the similarity as a team.

\subsection{Qualitative Data}

While the presented quantitative analysis reveals how and where teams perform differently in comparison to solo workers, the questionnaire responses, team interviews and researcher observations enrich our understanding of collocated crowdsourcing.

\section{Contrasting Collocated and Traditional Crowdsourcing}

One of the most profound differences that participants experienced between completing tasks alone with a desktop, i.e., the traditional crowdsourcing way, and completing same tasks as a team using TeamSourcer was the perceived amusement and enjoyment of teamwork. The questionnaires that the participants completed after the study revealed insights such as:

"For sure more fun, being able to discuss the familiar features or suspicious things and more exciting and dynamic for the Waldo one! It takes a bit more of time to complete the tasks, but it is more enjoyable",

"I think it was more funny thing doing in a team, while sitting alone I was more critical",

"I found very pleasant working as a team because we laugh and although some people had different ideas, everything was accepted. A consequence could be that my answer could be right and nobody agreed", and

"[We interacted] in a funny mode, laughing and discussing." 
This was further confirmed in the final team interviews, where several teams stated that teamwork was more fun than working solo. In addition, we observed all teams exhibited laughter -- including those that consisted of strangers. Particularly the social aspects of being in the same physical location were considered as a positive element by many participants:

"I do like interaction with people, so for me being closer to people in real life is much better. I can talk, and communicate and see their face and those things", or

"It is more fun when doing it in the same location"

So, and as expected, the natural human contact and interaction adds a touch of fun to otherwise "serious" work, which was also stated by the participants. Regarding interaction with other collocated participants, we observed various styles and techniques. During teamwork, participants were gesturing, pointing, and highlighting e.g. facial features using not only their own hands but also using their own faces. Further, participants could read subtle cues from others' work and verify others' participation in the joint effort:

"I could kind of see from the edge of my eye when someone found the Waldo",

"It can help you can feel the body language or if someone is a little bit shy or not saying things", or

"Now that we are together you at least know that others are working. If online, you don't know, they might be doing something else"

In the final interviews and especially during observations it became clear that non-verbal communication played a key role in helping the teams collaborate. The "Where's Waldo" task elicited excitement from the participants, which was sometimes followed by physical forms of encouragement from the other team members (e.g., patting the shoulder of the person who completed the task). Leaders often displayed an interesting behavior pattern, where they looked around the table before making a submission. We hypothesize that this was done to verify that all team members had completed their contribution to the task, and to assess whether the team was ready to move on to the next task.

In addition to enjoyment and ease of collaboration, several participants noted that especially during $\mathrm{T} 1$ and $\mathrm{T} 3$ there a lot of debate and reasoning took place before the team arrived to a conclusion about what to submit as the final answer. We also attempted to find out if the workers themselves think that teams produce higher quality, or faster, output than individuals. In this regard, the final verdict is unclear, with some participants favoring teamwork and others solo work:

"We were complimenting each other's choices and more eyes were looking to the images, so a better conclusion was made for every image as a team”,

"Working as a team was faster for Waldo one, but for the suspicious it was a bit controversial as I didn't seem to find many suspicious things on the images and the team mates were spotting quite a few", 
"It takes less time to complete the task alone. However, when working as a team new insights/problem solutions can be found. Feedback/opinions can be collected while working as a team or alone",

"I think it is more fun when working in a team, because you can see the questions from different perspectives and think about the questions in a brand new way”,

"Alone I didn't have to think what others would think of my decisions, so I could answer all questions freely", and

"Things that require some personal point view (as the suspicious one) can trigger some quite interesting debate or discussion but also raise some awareness with other's people point of view, while on finding Waldo it can for sure improve the overall performance (spotting something faster). When it comes to some collaborative work (as the family) it is good to receive other people's points of view and also try to argue and propose some of your own perspective."

When asked about the use of our setup for different types of tasks, most participants agreed that the collaborative approach will produce better and faster results. The negative comments about the setup focused on "wasting time" when debating something with the team:

"For performance (speed, accuracy), collocated work is better",

"It [collaborative] takes a bit more of time to complete the tasks, but it is more enjoyable",

"When I could not find it quickly someone else found it quickly so it brings out the best in everybody",

"If you want really fast [output], three different tasks [is better than a shared collaborative task]. But if you need to discuss, a shared canvas might be better",

"More time taken to make a decision when working as a group, yet more fun",

"Teamwork might not be effective in terms of managing resources to be committed if action was to be taken based on the activity that was done by the team (more people means more opinions on the table...)" and

"...it takes time to discuss with other, and if nothing comes out or topic goes to wrong direction, it could be just waste of time."

\section{Emerging Task Strategies}

Collocated crowdsourcing affords rich verbal and nonverbal interaction to take place among the workers. We observed several different strategies emerge during the study. 
Task 1 - Identify Suspicious Elements. Based on our observations, teams did not agree on a specific strategy for T1 prior to starting the task, but instead discussed their ideas as they were investigating the first few images. In several cases the participants agreed to others' suggestions without much discussion, resulting in submissions that consisted simply of a combination of the three individual's opinions with no filtering. However, a more common case was where debates took place and ultimately the leader had to make a verdict:

"For the suspicious everyone did their own thing. So when we submitted all the opinions were there",

"We discussed our decisions before making them (task 1 and 3) and towards the end we started to interact with the pictures individually before making the decision. In the beginning only the leader marked and submitted the answers",

"We discussed about the subject and try to make the best decision based on majority opinions",

"Yes, I am leader and sometimes I made the final decision if we disagree", or

The strategies of individual members such as starting with dangerous looking items, identifying people that appear unnatural to the context or noticing out-of-place facial expressions of people were then merged into team strategies. In addition, participants expressed hesitation when marking objects or people as suspicious, often adding extra information when explaining their choices to their team members. Other participants asked for confirmation from others before making a decision to mark someone as suspicious:

"You think this one?" - "Yes! Absolutely" - "I am also a bit worried",

"In my overall experience I wasn't finding many things suspicious. So if he told me that it was suspicious I thought - yea it can look suspicious" and

“...when people start pointing out things you notice them as well."

This behavior, in which participants are 'testing the water', allows participants to get an understanding on the group's collective opinion - after which they can more safely express their own thoughts (Noelle-Neumann, 1974).

Task 2 - Where's Waldo. Prior to the start of T2, we repeatedly explained to the participants that the fastest team would receive two additional movie vouchers. This primed the team to complete the task as fast as possible, which in some cases led to a detailed strategy discussion prior to starting the task. Several teams decided to divide the various areas of the image among the three members:

"Ok, should one go from one side. And the other one from the other side" - "Ok, so we split the screen like this" - "Ok so you go from left to right. And you from right to left, and then you'll go from up to down?" - "I'll check the middle." 
Other teams simply decided to go without any detailed strategy and to just encourage everyone to do their best. The difference in strategy also led to interesting observations. For a team consisting of strangers, when a participant announced they found Waldo, the leader looked on the screen to see where. The participant was somewhat hesitant to draw on an area that was not theirs, according to the agreed upon screen division tactic.

In general, as this was a competitive task, participants were experiencing more pressure to increase team performance. This resulted in a more hectic collaboration, where participants raised their voices in excitement, motivated their teammates through expressive gestures, and exclaimed loudly when they had found Waldo in the image. We observed this type of behavior across all teams regardless of their familiarity (friends / strangers).

Task 3 - Kinship. T3 resulted in the most collaboration of all tasks. Individual features were discussed in detail, before a decision was made to mark the two individuals in the photo as either family related or unrelated. The task did not have an obvious correct or wrong answer (as with T1), yet was less ambiguous than T1, because T3 actually had a ground truth available to us. Thus, participants provided extensive effort to explain their choices to their teammates while working on the task:

"I tried to show why I think something, so others knew what I was doing",

"Look at their nose” - "No, man, look at her nose, and his nose. Hers is pointy and [...]".

Participants were also willing to accept the decision of other team members, which is similar to the observed behavior in T1. A critical difference is that T3 required a specific boolean decision to be made (kinship or no). Participants could therefore not always accept the input of their teammates if they did not reach agreement. This sometimes resulted in situations in which participants were forced to follow the majority vote even though they did not necessarily fully agree:

"I felt pressure by these guys. I had to follow the consensus, even if there are similarities it doesn't have to mean but..",

"Yea, everyone else said it is .. helps to go along with it" or

"In a group, I need to compromise sometimes"

Ultimately, it was clear that in T3 the teams naturally conversed and collaborated extensively and with great care. In most cases, this resulted in a joint effort to identify similar features and kinship status, regardless of the teams' prior familiarity with each other.

\section{Impact of Team Familiarity}

In the study, we formed teams that contained strangers to explore the impact of a priori familiarity. The interview and questionnaire administered following the completion of the workers' tasks asked participants to comment on the team dynamics in this regard. Typically, participants stated that familiarity made little or no difference in the working process, or that the members become familiar enough for this type of transient work very fast: 
"I don't think knowing each other changed the task",

"It was a bit hard at the beginning to communicate, as it went further, it was better" or

"At the beginning maybe [communication was affected], but after getting to know the person there were no issues."

The type of task also influenced the way in which our participants experienced different team compositions. In the case of T3, where which participants identified suspicious elements, no obvious answer is available and prejudices of individual participants might be more prevalent:

"If I had known the other members, I would have given more reasons as why I found someone suspicious... But now I applied a social filter" or

"I felt more comfortable when doing it alone. Because I did not always agree [on what was suspicious]."

In case of disagreement with their teammates, participants sometimes decided not to fight the majority decision. This happened with all teams:

"I just think anyone on them can be suspicious... So if they [teammates] think they are, sure" and

“What?! I don't agree but okay."

Others were however quick to point out areas of disagreement with their teammates:

"There are several guys with hats." - "Yes because they are cold."

Ultimately, the overarching conclusion is that completing the tasks was more important to the teams than letting familiarity or non-familiarity have an impact on the work. Unfamiliar teams had no problems interacting with each other after "breaking the ice", and both types of teams enjoyed solving the tasks together.

\section{Design Opportunities}

Our study revealed several issues to develop and explore further. In one of the discussions following the completion of the tasks, one participant offered the suggestion of using a single device rather than several:

"One tablet is maybe better than three. Everyone doing it on one device, bigger device."

One of his teammates disagreed, mentioning the fact that the current design allows for more people to freely express their opinion:

"If this happens on one screen - maybe some people might not express their opinion... So if we have three tablets we can be sure that everyone mentions their opinion." 
A similar point of view was heard from other participants, for example:

"I like the fact that it is a round table, because you can see each other faces. It facilitates conversation, a big screen would be worse. You cannot experience the feelings of people etcetera. I also kind of like that everyone has their own screen."

We observed participants leaning in on the tablets of team members, either to point out something they observed, or to get an understanding of what their co-worker was talking about. One participant suggested that the tablet software could offer support for this kind of activity that is less destructive and time consuming than drawing and erasing on the image.

"I guess you could implement a short suggest option. [...] You could do this like a highlight, so people could see where your finger is. So you are not committed, but people still see what you do."

Finally, another participant using different colors for each team member:

"Maybe different colours for each member. We would kind of feel entitled to what we mark, and don't necessarily feel that we have to agree what others say."

\section{DISCUSSION}

\subsection{Benefits of Collocated Interaction in Crowdsourcing}

Our findings identify a number of benefits in the deployed approach, i.e., teamwork in situated crowdsourcing. First, participants expressed how completing the tasks as a team in the same physical location made the tasks feel less like a "chore" and more fun. Previous work has shown that overall enjoyment when completing a task leads to increased levels of productivity (Oswald et al., 2009), meaning that happy crowdsourcing workers are more likely to complete tasks more efficiently. While this can be achieved with the design of the crowdsourcing task itself (e.g., gamification), in a scenario where the tasks are equal, enjoyment is more likely to be higher in teams.

Second, the collocated nature of our experiment facilitated conversation and interaction between participants, which positively impacted how the crowdsourcing work was conducted. The benefits of mediating teamwork with technology deployments, of course, have been shown previously on several HCI and CSCW related domains, such as sensemaking (Wozniak et al., 2016), brainstorming (Lucero et al., 2010) or online crowdsourcing (Niloufar et al., 2017). In our case and in contrast to online teambased crowdsourcing, several participants stated how performing the same tasks with a team online would not allow them to ensure that the other members were actually engaged with the task, as it would be difficult to see what they are doing. Even if the remote team member's screen was shown to other workers (remote desktop style solutions have been studied earlier), the participant might still be using a mobile phone or be otherwise not focusing to the task at hands. When working in a collocated fashion, it is easy to perceive the other workers' state and motivation to work for a common good.

The participants naturally discussed and gathered input from all the members (verbally and through body language). For the most part, our participants practiced caution and conversed and debated their 
decisions before jumping into conclusions. This resulted in significantly longer completion times for T1 and T3 (Figure 7) than solo workers. However, teams completed T2 faster than solo workers since it was an objective task that has only 1 correct answer. By having two extra pairs of eyes surveying the image at a given time resulted in faster completion times, but not necessarily improved accuracy. In general, teams were able to reach a consensus, or in some cases (especially with T1, T3) resorted to using a majority voting scheme. Agreement filters are a commonly used method in crowdsourcing to remove unwanted contributions (Bernstein et al., 2010). In our case, the same notion is applied already in the contribution stage when the teams are working and at the same time interacting to arrive to a conclusion about the outcome. We believe our findings in this regard can inform the design of teambased online crowdsourcing platforms. In online teamwork environments, it could be helpful to implement collaboration mechanisms that focus also on person-to-person communication instead of enabling only collaboration focusing on the task.

\subsection{Challenges in Teamwork}

Collocated crowdsourcing is not without its drawbacks, and in other teamwork contexts the tradeoff between personal satisfaction and the group contribution quality has been highlighted (Desanctis \& Gallupe, 1987). Reflecting on T1, participants described how they easily swayed their opinions and thoughts when one of their team members expressed an opinion on suspicious behavior. Related to this, there is an interesting real-world link to the 2013 bombing event in Boston (which was in fact the original motivation for the design of T1). While the success of the bombing event's investigation was largely attributed to crowdsourcing in the media, a less-advertised side to the story is how many people started to believe false statements said by others online. The typical visual search task then "quickly morphed into a much uglier digital witch hunt, one where the crowd's fears, prejudices, and suspicions were given credence to crowdsource investigations turned into ugly digital witch-hunt", as articulated by Forbes (Lessons From Crowdsourcing the Boston Bombing Investigation, 2016). This aspect of crowdsourcing-gone-wrong led to many innocents being harassed by self-proclaimed vigilantes both online and offline, and exemplifies how flawed the group's collective judgement when social influence comes into play (Surowiecki, 2005).

Social influence was indeed observed in our study as well. Participants unsure about their verdict of the task at hands quickly either let others do the work or looked for other cues among the team members. These observations are also in line with the theory on "The Spiral of Silence" that describes the interaction between an individual and the surrounding group especially when discussing subjective opinions (Noelle-Neumann, 1974). Individuals adjust their behavior (e.g., willingness to express their opinion) to follow the dynamics of the conversation and to support the prevailing argument(s) of the group discussion. Further, this happens particularly amongst strangers. As a result, individuals are less eager to express themselves when their opinion is not in line with the current common opinion of the group; "to the individual, not isolating himself is more important than his own judgement" (NoelleNeumann, 1974). We believe this is an important future aspect to study and attempt to mitigate in collocated crowdsourcing.

Finally, another potential issue is when teams do not reach a consensus, and "get stuck" on a task. This can result in extremely long task completion times as well as degrading working environment. In our case the design choice of having a leader, informed by work in (Retelny et al., 2014), worked well in mitigating this issue. For instance, in T1 several teams ended up debating and disagreeing about the subjective result, and a leader was a necessity for a decision to be made. The strategy on how to choose 
a leader can however be debated. In a collocated setting such as ours, the first team member to start a session feels like a natural choice for a leader, but other ways, such as changing the leader on the go when a more seasoned worker based on task performance history in the same market joins the session, can be explored in the future.

\subsection{Does Team Familiarity Affect Output?}

It is reasonable to assume that familiarity can in some cases play an important role in collocated team-based crowdsourcing. We aimed to investigate what role do social relationships play in the emerging interactions of a team performing crowdsourcing together. Familiarity of teams in crowdsourcing is a relatively unexplored concept, and it has mostly been explored with online workers and by implementing computational systems that recruit the workers directly from labor markets (Niloufar et al., 2017). In situated crowdsourcing, however, especially the availability of workers is entirely different than in online crowdsourcing (Hosio et al., 2014). One cannot just tap into a local labor market and have a stream of situated workers ready to join and form teams. Instead, the availability and the potential familiarity of the workers is defined by the space and context around the deployment.

In this regard, the role of the assigned team leader will become even more prominent than online: the leader will assemble the team of either friends or attempt to get strangers involved in work. In our experiment, it was seen as fun to work with a team -- friends or not -- but especially with friends, interaction was seen as natural and comfortable. Will more comfortable interaction lead to better quality output? That, we cannot answer with certainty. Teams were confident of achieving better output quality together rather than alone, but also some members acknowledged how they must make compromises to satisfy the team's need for consensus, or just be content with majority vote. It is only fair to assume that familiarity will have a role in such considerations. In time-critical cases, we believe, it could be detrimental to have friends only in a team, as the team will not necessarily focus on the work at hands and are driven by a very relaxed social atmosphere. On the other hand, enabling a great working environment is important as well, and in crowdsourcing the focus is often too much on simply producing labor without considering working conditions. We will set to investigate this in our followup in-the-wild deployments with TeamSourcer.

\subsection{Research Opportunities}

More than in the physical design of a platform such as ours, we believe the potential for important future research on this domain lies in studying what type of tasks would benefit from collocated work. We collected ample evidence on how collocated work fosters interaction, and the next step is to explore which tasks can be cost-effectively completed in teams so that the results warrant the use of such worker groups. Team spirit and completing tasks together can clearly make collocated crowdsourcing an enjoyable experience, and the impact of this on, for example, payment schemes, is a good starting point for further investigations.

Second, team size is an interesting variable to explore in our future deployments. The current setup limits the number of simultaneous collocated workers to three. TeamSourcer's application, however, has no upper limit to team size, and simply deploying more devices allows larger teams to work at the same time. Further, the members do not need to even necessarily all be collocated, but some can contribute remotely, as long as the tasks provide support for this. Then, attracting users to a team 
becomes an issue. We assume it is the team leader's task to find people to work with - and with a disruptive situated deployment this should not be difficult (see e.g. (Goncalves et al., 2013)).

Finally, as the TeamSourcer platform itself has been designed to be generic enough to support many types of tasks (tasks are responsible for enforcing or not enforcing collaboration), we will open up the market for local 3rd parties and research units to pitch in creative tasks. With Huddler (Niloufar et al., 2017), for example, online teams were shown to excel in creative tasks, and collocated interaction may very well prove as excellent catalyst for providing even higher quality creative output. For this, however, we must first implement an online portal to manage the tasks and output, much like what traditional labor markets online provide.

\title{
6.5. Limitations
}

We acknowledge certain limitation in the presented work. First, the statistical analysis regarding completion time in all tasks was implemented using client side JavaScript timers. However, the used collaborative canvas solution (awwapp.com) does not feature a callback after a new image has been loaded on the canvas. Therefore, we were required to start the timer for a new task instance upon calling AWWApp's API to load the next image(s). In some cases, we noticed a lag when workers were working as teams. However, this issue affects mostly T2, as it was designed as a competition about completion times, and as teamwork was found faster, eliminating the lag would just strengthen the effect.

Second, we cannot generalize the results to all cultures and locations. Especially with collocated interaction we believe cultures, ages, tech backgrounds etc. play a major role. However, our work is concerned with developing crowdsourcing and contributing a new type of artifact and study to the domain. Therefore, we argue that our results provide a good starting point for developing further and discussing the potential and use cases of collocated crowdsourcing.

\section{CONCLUSION}

In this paper, we presented the design and initial experiences with a novel crowdsourcing system: TeamSourcer. By design, it encourages natural interaction between collocated workers. While technically the member count of teams is not limited, in this study we used it for groups of 3 workers. We found that the workers greatly enjoyed working as a team when compared to traditional crowdsourcing. We report on the strategies that collocated interaction enables for crowdsourcing tasks. Team familiarity did not seem to have much effect on task output quality, and workers became friendly with each other rather fast when working together. Our study is the first to focus solely on collocated crowdsourcing in teams and highlights a wide variety of new research opportunities. As also suggested by Kittur in the article Crowdsourcing, Collaboration and Creativity (Kittur, 2010), we must learn to harness the innate power of groups and teamwork in order to take crowdsourcing the next level.

\section{REFERENCES}

\author{
Alt, F., Shirazi, A. S., Schmidt, A., Kramer, U., Nawaz, Z. (2010). Location-based crowdsourcing: \\ Extending crowdsourcing to the real world. NordiCHI '10: NordiCHI. ACM.
}


Anslow, C., Campos, P., Grisoni, L., Lucero, A. (2015). Collaboration meets interactive surfaces (CMIS): Walls, tables, mobiles, and wearables. ITS '15: Proceedings of the 2015 international conference on interactive tabletops \& surfaces. ACM.

Bernstein, M. S., Little, G., Miller, R. C., Hartmann, B., Ackerman, M. S., Karger, D. R., Crowell, D., Panovich, K. (2010). Soylent: A word processor with a crowd inside. UIST '10: Proceedings of the 23nd annual ACM symposium on user interface software and technology. ACM.

Bly, S. A., Harrison, S. R., Irwin, S. (1993). Media spaces: Bringing people together in a video, audio, and computing environment. Commun. ACM, 36, 28-46.

Boring, S., Jurmu, M., Butz, A. (2009). Scroll, tilt or move it: Using mobile phones to continuously control pointers on large public displays. OZCHI '09: Proceedings of the 21 st annual conference of the australian computer-human interaction special interest group: Design: Open 24/7. ACM.

Brignull, H., Rogers, Y. (2003). Enticing people to interact with large public displays in public spaces. Proceedings of INTERACT.

Clickworker. [Web page] Retrieved from http://www.clickworker.com on September 28, 2014.

Davies, N., Langheinrich, M., Jose, R., Schmidt, A. (2012). Open display networks: A communications medium for the 21 st century. Computer, $45,58-64$.

Desanctis, G., Gallupe, R. B. (1987). A foundation for the study of group decision support systems. Manage. Sci., 33, 589-609.

Farnham, S. D., McCarthy, J. F., Patel, Y., Ahuja, S., Norman, D., Hazlewood, W. R., Lind, J. (2009). Measuring the impact of third place attachment on the adoption of a place-based community technology. CHI '09: Proceedings of the SIGCHI conference on human factors in computing systems. ACM.

Fikkert, W., Vet, P. V. D., Veer, G. V. D., Nijholt, A. (2009). Gestures for large display control. Gesture in embodied communication and human-computer interaction. Springer Berlin Heidelberg.

Fischer, J. E., Reeves, S., Rodden, T., Reece, S., Ramchurn, S. D., Jones, D. (2015). Building a birds eye view: Collaborative work in disaster response. CHI 15: Proceedings of the 33rd annual ACM conference on human factors in computing systems. ACM.

Gehring, S., Krüger, A. (2012). Using media facades to engage social interaction. UbiComp '12: Proceedings of the 2012 ACM conference on ubiquitous computing. ACM.

Goncalves, J., Ferreira, D., Hosio, S., Liu, Y., Rogstadius, J., Kukka, H., Kostakos, V. (2013). Crowdsourcing on the spot: Altruistic use of public displays, feasibility, performance, and behaviours. UbiComp: International joint conference on pervasive and ubiquitous computing. ACM. 
Goncalves, J., Hosio, S., Kostakos, V. (2017). Eliciting structured knowledge from situated crowd markets. ACM Transactions on Internet Technology, online first .

Goncalves, J., Hosio, S., Rogstadius, J., Karapanos, E., Kostakos, V. (2015). Motivating participation and improving quality of contribution in ubiquitous crowdsourcing. Computer Networks, 90 , 34-48.

Goyal, N., Fussell, S. R. (2016). Effects of sensemaking translucence on distributed collaborative analysis. CSCW '16: Proceedings of the 19th ACM conference on computer-supported cooperative work \& social computing. ACM.

Gray, M. L., Suri, S., Ali, S. S., Kulkarni, D. (2016). The crowd is a collaborative network. CSCW '16: Proceedings of the 19th ACM conference on computer-supported cooperative work \& social computing. ACM.

Greenfield, A., Shepard, M. (2007). Situated technologies pamphlets 1: Urban computing and its discontents. The Architectural League of New York, New York.

Heimerl, K., Gawalt, B., Chen, K., Parikh, T., Hartmann, B. (2012). CommunitySourcing: Engaging local crowds to perform expert work via physical kiosks. Proceedings of the SIGCHI conference on human factors in computing systems.

Hole-In-Space. [Web page]. Retrieved from http://www.ecafe.com/getty/HIS/index.html on August 8, 2016.

Hosio, S., Goncalves, J., Anagnostopoulos, T., Kostakos, V. (2016). Leveraging wisdom of the crowd for decision support. BCS-HCI: British human computer interaction conference. ACM.

Hosio, S., Goncalves, J., Lehdonvirta, V., Ferreira, D., Kostakos, V. (2014). Situated crowdsourcing using a market model. UIST: User interface software and technology. ACM.

Hosio, S., Goncalves, J., van Berkel, N., Klakegg, S. (2016). Crowdsourcing situated \& subjective knowledge for decision support. UbiComp '16 Adjunct: International joint conference on pervasive and ubiquitous computing adjunct. ACM.

Hosio, S., Harper, R., O'Hara, K., Goncalves, J., Kostakos, V. (2015). Life through the lens: A qualitative investigation of human behaviour with an urban photography service. $B C S$ - $H C I$ : British human computer interaction conference. ACM.

Hosio, S., Jurmu, M., Kukka, H., Riekki, J., Ojala, T. (2010). Supporting distributed private and public user interfaces in urban environments. HotMobile '10: Proceedings of the eleventh workshop on mobile computing systems \& applications. ACM.

Hosio, S., Kostakos, V., Kukka, H., Jurmu, M., Riekki, J., Ojala, T. (2012). From school food to skate parks in a few clicks: Using public displays to bootstrap civic engagement of the young. Pervasive: International conference on pervasive computing. Springer. 
Hosio, S., Kukka, H., Goncalves, J., Kostakos, V., Ojala, T. (2016). Toward meaningful engagement with pervasive displays. IEEE Pervasive Computing, 15, 24-31.

Hosio, S., Kukka, H., Riekki, J. (2010). Social surroundings: Bridging the virtual and physical divide. IEEE MultiMedia, , 26-33.

Huang, E. M., Mynatt, E. D., Trimble, J. P. (2007). When design just isn't enough: The unanticipated challenges of the real world for large collaborative displays. Personal Ubiquitous Comput., 11, 537-547.

Huang, Y. -C. (2015). Designing a micro-volunteering platform for situated crowdsourcing. CSCW'15 Companion: Proceedings of the 18th ACM conference companion on computer supported cooperative work \&\#38; social computing. ACM.

Ipeirotis, P. G., Gabrilovich, E. (2014). Quizz: Targeted crowdsourcing with a billion (potential) users. Proceedings of the 23rd international conference on world wide web.

José, R., Otero, N., Izadi, S., Harper, R. (2008). Instant places: Using bluetooth for situated interaction in public displays. IEEE Pervasive Computing, 7, 52-57.

Kagel, J. H., Roth, A. E. (1995). The handbook of experimental economics. Princeton, N.J.: Princeton University Press.

KinFaceW: Kinship Face in the Wild Database. [Web page]. Retrieved from http://www.kinfacew.com on October 13, 2016.

Kittur, A. (2010). Crowdsourcing, collaboration and creativity. XRDS, 17, 22-26.

Kittur, A., Nickerson, J. V., Bernstein, M., Gerber, E., Shaw, A., Zimmerman, J., Lease, M., Horton, J. (2013). The future of crowd work. CSCW'13: Proceedings of the 2013 conference on computer supported cooperative work. ACM.

Kuikkaniemi, K., Jacucci, G., Turpeinen, M., Hoggan, E., Müller, J. (2011). From space to stage: How interactive screens will change urban life. Computer, 44, 40-47.

Kukka, H., Oja, H., Kostakos, V., Goncalves, J., Ojala, T. (2013). What makes you click: Exploring visual signals to entice interaction on public displays. CHI: Conference on human factors in computing systems. ACM.

Lessons From Crowdsourcing the Boston Bombing Investigation. [Web page]. Retrieved from http://www.forbes .com/sites/tarunwadhwa/2013/04/22/lessons-from-crowdsourcing-the-bostonmarathon-bombings-investigation/\#199d9b2212b5 on September 26, 2016.

Lucero, A., Keränen, J., Korhonen, H. (2010). Collaborative use of mobile phones for brainstorming. MobileHCI '10: Proceedings of the 12th international conference on human computer interaction with mobile devices and services. ACM. 
Ludwig, T., Kotthaus, C., Reuter, C., Dongen, S. v., Pipek, V. (2016). Situated crowdsourcing during disasters: Managing the tasks of spontaneous volunteers through public displays. International Journal of Human-Computer Studies.

Lykourentzou, I., Kraut, R. E., Dow, S. P. (2017). Team dating leads to better online ad hoc collaborations. CSCW '17: Proceedings of the 2017 ACM conference on computer supported cooperative work and social computing. ACM.

Memarovic, N., Elhart, I., Rubegni, E. (2016). Developing a networked public display system. IEEE Pervasive Computing, 15, 32-39.

Memarovic, N., Langheinrich, M., Alt, F., Elhart, I., Hosio, S., Rubegni, E. (2012). Using public displays to stimulate passive engagement, active engagement, and discovery in public spaces. MAB: Media architecture biennale conference. ACM.

Memarovic, N., Langheinrich, M., Cheverst, K., Taylor, N., Alt, F. (2013). P-LAYERS -- A layered framework addressing the multifaceted issues facing community-supporting public display deployments. ACM Trans. Comput.-Hum. Interact., 20, 17:1-17:34.

Morris, R. R., Dontcheva, M., Gerber, E. M. (2012). Priming for better performance in microtask crowdsourcing environments. Internet Computing, IEEE, 16, 13-19.

Müller, J., Alt, F., Michelis, D., Schmidt, A. (2010). Requirements and design space for interactive public displays. MM '10: Proceedings of the international conference on multimedia. ACM.

Müller, J., Eberle, D., Tollmar, K. (2014). Communiplay: A field study of a public display mediaspace. CHI '14: Proceedings of the 32nd annual ACM conference on human factors in computing systems. ACM.

Niloufar, S., Andrew, M., Melissa, V., Michael, B. (2017). Huddler: Convening stable and familiar crowd teams despite unpredictable availability. ACM Conference on Computer-Supported Cooperative Work and Social Computing : .

Noelle- Neumann, E. (1974). The spiral of silence - A theory of public opinion. Journal of Communication, 24, 43-51.

Ojala, T., Kostakos, V., Kukka, H., Heikkinen, T., Linden, T., Jurmu, M., Hosio, S., Kruger, F., Zanni, D. (2012). Multipurpose interactive public displays in the wild: Three years later. Computer, 45 , 42-49.

Oswald, A. J., Proto, E., Sgroi, D. (2009). Happiness and productivity. IZA Discussion Paper No. 4645.

Peltonen, P., Kurvinen, E., Salovaara, A., Jacucci, G., Ilmonen, T., Evans, J., Oulasvirta, A., Saarikko, P. (2008). It's mine, don't touch!: Interactions at a large multi-touch display in a city centre. $\mathrm{CHI}$ '08: $\mathrm{CHI}$. 
Poncela-Casasnovas, J., Gutiérrez-Roig, M., Gracia-Lázaro, C., Vicens, J., Gómez-Gardeñes, J., Perelló, J., Moreno, Y., Duch, J., Sánchez, A. (2016). Humans display a reduced set of consistent behavioral phenotypes in dyadic games. Science Advances, 2, e1600451.

Porcheron, M., Lucero, A., Fischer, J. E. (2016). Co-curator: Designing for mobile ideation in groups. AcademicMindtrek '16: Proceedings of the 20th international academic mindtrek conference. ACM.

Reagans, R., Argote, L., Brooks, D. (2005). Individual experience and experience working together: Predicting learning rates from knowing who knows what and knowing how to work together. Manage. Sci., 51, 869-881.

Retelny, D., Robaszkiewicz, S., To, A., Lasecki, W. S., Patel, J., Rahmati, N., Doshi, T., Valentine, M., Bernstein, M. S. (2014). Expert crowdsourcing with flash teams. UIST '14: Proceedings of the 27th annual ACM symposium on user interface software and technology. ACM.

Sasao, T., Konomi, S., Suzuki, R. (2016). Supporting community-centric use and management of vacant houses: A crowdsourcing-based approach. UbiComp '16: Proceedings of the 2016 ACM international joint conference on pervasive and ubiquitous computing: Adjunct. ACM.

Scheible, J., Ojala, T. (2009). MobiSpray: Mobile phone as virtual spray can for painting BIG anytime anywhere on anything. SIGGRAPH '09: ACM SIGGRAPH 2009 art gallery. ACM.

Schroeter, R., Foth, M., Satchell, C. (2012). People, content, location: Sweet spotting urban screens for situated engagement. DIS '12: Proceedings of the designing interactive systems conference. ACM.

Scott, S. D., Carpendale, M. S. T., Inkpen, K. M. (2004). Territoriality in collaborative tabletop workspaces. CSCW '04: Proceedings of the 2004 ACM conference on computer supported cooperative work. ACM.

Shen, C. E., Ryall, K., Kathleen. (2003). UbiTable: Impromptu face-to-face collaboration on horizontal interactive surfaces. A. K. S. A. M. J. F. Dey (Ed.), UbiComp 2003: Ubiquitous computing: 5th international conference, seattle, WA, USA, october 12-15, 2003. Proceedings. Berlin, Heidelberg: Springer Berlin Heidelberg.

Smith, A. M., Lynn, S., Lintott, C. J. (2013). An introduction to the zooniverse. First AAAI conference on human computation and crowdsourcing.

Storz, O., Friday, A., Davies, N., Finney, J., Sas, C., Sheridan, J. (2006). Public ubiquitous computing systems: Lessons from the e-campus display deployments. IEEE Pervasive Computing, , 40-47.

Surowiecki, J. (2005). The wisdom of crowds: Why the many are smarter than the few and how collective wisdom shapes business, economies, societies and nations. New York: Anchor.

Taylor, N., Cheverst, K. (2012). Supporting community awareness with interactive displays. Computer, , 26-32. 
Terrenghi, L. Q., Dix, A., Alan. (2009). A taxonomy for and analysis of multi-person-display ecosystems. Personal and Ubiquitous Computing, 13, 583.

Tomnod. [Web page]. Retrieved from http://www.tomnod.com on September 26, 2016.

Weber, R. P. (1990). Basic content analysis. Newbury Park, Calif.: Sage Publications.

A Web Whiteboard. [Web page]. Retrieved from https://awwapp.com on September 26, 2016.

Weiser, M. (1999). The computer for the 21 st century. ACM SIGMOBILE Mobile Computing and Communications Review, 3, 3-11.

Weißker, T., Berst, A., Hartmann, J., Echtler, F. (2016). The massive mobile multiuser framework: Enabling ad-hoc realtime interaction on public displays with mobile devices. PerDis '16: Proceedings of the 5th ACM international symposium on pervasive displays. ACM.

Wozniak, P., Goyal, N., Kucharski, P., Lischke, L., Mayer, S., Fjeld, M. (2016). RAMPARTS: Supporting sensemaking with spatially-aware mobile interactions. CHI '16: Proceedings of the 2016 CHI conference on human factors in computing systems. ACM. 